\title{
EFFECT OF PHOSPHORUS ON N, P, K, Mg ACCUMULATION AND PLANT GROWTH OF DIFFERENT CITRUS ROOTSTOCKS
}

\author{
Tu, P. F. ${ }^{1,2,4,5 \dagger}-$ DENG, L. S. ${ }^{2 \dagger}-$ LI, J. ${ }^{3}-$ ZHANG, C. L. ${ }^{2}-$ HE, S. X. ${ }^{1}-$ CHEN, J. Z. ${ }^{1 *}-$ \\ CHENG, F. X. ${ }^{4}-$ JI, J. H. ${ }^{5}$ \\ ${ }^{1}$ College of Horticulture, South China Agricultural University \\ Guangzhou 501642, China \\ ${ }^{2}$ College of Natural Resources and Environment, South China Agricultural University \\ Guangzhou 501642, China \\ ${ }^{3}$ Department of Horticulture, Zhongkai University of Agriculture and Engineering \\ Guangzhou, China \\ ${ }^{4}$ Guangzhou Yixiang Agriculture Technology Co. Ltd. \\ Guangzhou 501642, China \\ ${ }^{5}$ Dongguan Yixiang Liquid Fertilizer Co. Ltd. \\ Dongguan 523135, China \\ *Corresponding author \\ e-mail: cjzlxb@scau.edu.cn
}

${ }^{\dagger}$ These authors have contributed equally to this work.

(Received $11^{\text {th }}$ Aug 2017; accepted $11^{\text {th }}$ Jan 2018)

\begin{abstract}
Phosphorus is an essential nutrient for the growth of citrus plants. A pot experiment was conducted in greenhouse to investigate the citrus cultivars grafted on 10 rootstocks (Cuningmeng, Suanju, Suancheng, Honglimeng, Zhike, Goutoucheng, Xiangyuan, Hongju, Xiangcheng, and Zhicheng) in response to the different phosphorus treatments viz, low $\mathrm{P}(\mathrm{LP})=0.01 \mathrm{mmol} \mathrm{L}^{-1}$, moderate $\mathrm{P}(\mathrm{MP})=0.5$ mmol L $\mathrm{L}^{-1}$, high $\mathrm{P}(\mathrm{HP})=1 \mathrm{mmol} \mathrm{L} \mathrm{L}^{-1}$ ) concentrations. All $\mathrm{P}$ concentrations were employed in pots containing Hoagland solution. The leaves, branch, root, and total dry weight, and nitrogen, phosphorus, potassium and magnesium concentrations in the different plant parts were determined. Results showed that $\mathrm{P}$ application increased $\mathrm{N}, \mathrm{P}, \mathrm{K}$ and $\mathrm{Mg}$ content in different plant parts. With increasing $\mathrm{P}$ application, the root dry weight, branch dry weight, leaves dry weight, and total dry weight increased whilst low $\mathrm{P}$ treatment affected the $\mathrm{P}, \mathrm{K}$, and $\mathrm{Mg}$ accumulation in root and ultimately plant growth. Moreover, low $\mathrm{P}$ concentrations also affected $\mathrm{Mg}$ content in leaves, that can lead to low plant photosynthesis and finally decreased the plant dry biomass

Keywords: citrus rootstocks, phosphorus treatment, plant growth, plant nutrients accumulation
\end{abstract}

\section{Introduction}

The rootstocks have a critical role in the fruit cultivation (Shafieizargar et al., 2012; Cantuarias-Avilés et al., 2010, 2011; Tazima et al., 2013). Rootstock affects the horticultural characteristics in citrus, such nutritional quality (Zhang et al., 2011), tree performance (Cantuarias-Avilés et al., 2010), stress resistance (Simpson et al., 2014), fruit yield (Hussain et al., 2013), and quality (Benjamin et al., 2013). Thus, it is meaningful to choose various rootstocks for investigating citrus plant growth, elements absorption, yield, quality; however, studies are still needed to evaluate the adopted rootstocks for citrus trees. 
Phosphorus is important for plant growth, development and reproduction for it is constituent of phospholipids, nucleic acid and many proteins. Low $\mathrm{P}$ availability in soil is one of the most critical aspects that limit the productivity of many crops (Sanchez and Salinas, 1981; Liao and Yan, 2003). P deficiency or excess could cause nutritional imbalance in citrus tree, then may reduce the yield and fruit quality (Fan and Wang, 2012; Fan and Luo, 2015). Too low or too high P concentrations may disturb the uptake of N, K, Fe, Zn, and B in plants (Fan and Wang, 2012). P also affects the root morphological and physiological characteristics of different root stocks (Fan and Luo, 2015). On the other hand, Syvertsen (1987) did not find any relationship between the $P$ level in leaves and photosynthetic capacity of orange and pomelo seedlings. Moreover, Bernardi et al. (2015) reported that $\mathrm{P}$ had little effects on photosynthesis. Zambrosi et al. (2013a) indicated that $\mathrm{P}$ uptake by young citrus plants in low $\mathrm{P}$ soil depends on rootstock varieties and nutrient management. Significant variations in the ability of citrus rootstocks to acquire $\mathrm{P}$ from the soil was differs with the nature of the rootstock (Wutscher, 1989; Mattos et al., 2006). Additionally, P application could mitigate the effects of hostile environment (Pestana et al., 2005; Gimeno et al., 2010). Zambrosi et al. (2013b) stated that sufficient $\mathrm{P}$ might contribute to increase the ability of young citrus trees to cope with $\mathrm{Cu}$ toxicity. Hence, $\mathrm{P}$ application could alter morphophysiological characteristics, yield and quality and nutrient uptake in plants, however, there are still some specific mechanisms that explain the differential responses of cultivated citrus to $\mathrm{P}$ fertilization are not yet fully explored. The specific objective of this study was to evaluate the effect of the $\mathrm{P}$ on nutritional accumulation and citrus tree growth.

\section{Materials and Methods}

\section{Experimental design}

A pot experiment was conducted in greenhouse in South China Agricultural University. Citrus cultivars Cuningmeng (Citrus jambhiri Lush), Suanju (Citrus reticulata Blanco), Suancheng, Honglimeng (Citrus limonia Osbeck), Zhike [Poncirus trifoliate (L.) Raf.], Goutoucheng (Citrus aurantium L.), Xiangyuan (Citrus medica var - ethrog Engl. ), Hongju (Citrus reticulata Blanco cv. Red tangerine), Xiangcheng (Citrus junons Sieb.), and Zhicheng (hybird of Citrus sinensis Osbeck. $\times$ Poncirus trifoliate Raf) were used as rootstocks in this study. Five leaf old seedlings were transferred to the different $\mathrm{P}$ treatments in Hoagland solution. Three $\mathrm{P}$ treatments viz, low $\mathrm{P}$ treatment $(\mathrm{LP})=0.01 \mathrm{mmol} \mathrm{L}^{-1}$, moderate $\mathrm{P}$ treatment $(\mathrm{MP})=0.5 \mathrm{mmol} \mathrm{L}^{-1}$, high $\mathrm{P}$ treatment $(\mathrm{HP})=1 \mathrm{mmol} \mathrm{L}^{-1}$ ) were employed in Hoagland solution with four replications. The nutrient solution was renewed once every three days.

\section{Sampling and measurement}

\section{Dry weight measurement}

After 45 days of treatment, three representative plants in each treatment and each cultivar were harvested and separated into root, branch and leaves. The plant samples were then oven dried at $105^{\circ} \mathrm{C}$ for $30 \mathrm{~min}$ and then at $75^{\circ} \mathrm{C}$ to a constant weight for measurement of dry weight. 


\section{$N, P, K$ and $M g$ content measurements}

The plant root, branch and leaves sample were then ground into powder for measurement of $\mathrm{N}, \mathrm{P}, \mathrm{K}$ and $\mathrm{Mg}$ content and calculation of $\mathrm{N}, \mathrm{P}, \mathrm{K}$ and $\mathrm{Mg}$ accumulation.

The $\mathrm{N}, \mathrm{P}$, and $\mathrm{K}$ content in plant root, branch and leaves were determined as described by $\mathrm{Lu}$ (1999). For $\mathrm{N}, \mathrm{P}$ and $\mathrm{K}$ contents, the dried samples ( 0.3 g) were digested using the $\mathrm{H}_{2} \mathrm{SO}_{4}-\mathrm{HClO}_{4}$ method. The digestion mixtures were then used to determine the total $\mathrm{N}$ content by the Kjeldahl method with a 2300 Kjeltec Analyzer Unit (Foss Tecator AB, Sweden), total $\mathrm{P}$ concentration by using a spectrophotometer (Shimadzu UV-2550), and total K concentration by atomic absorption spectrometry (SHIMADZU AA- 6300C AA spectrometer).

Dry sample of plant root, branch and leaves were ground, and approximately $0.100 \mathrm{~g}$ of the plant samples were dry-digested in a muffle furnace at $500^{\circ} \mathrm{C}$ for $6 \mathrm{~h}$, and then 10 $\mathrm{ml}$ of $\mathrm{HNO}_{3}: \mathrm{H}_{2} \mathrm{O}(1: 1)$ was added to extract the ions. The contents of concentration were detected by using an atomic absorption spectrometry (SHIMADZU AA- 6300C AA spectrometer). The $\mathrm{Mg}$ content in plant root, branch and leaves was recorded and expressed in $\mathrm{mg} \mathrm{g}^{-1}$.

\section{Statistical Analysis}

Analyses of variances (ANOVA) and correlation analyses were performed by Statistix version 8 (Statistix 8, Analystical, Tallahassee, FL, USA). Comparisons of means among different $\mathrm{P}$ treatments were made according to the least significant difference (LSD) test at 5\% probability level.

\section{Results}

\section{Citrus plant growth}

Significant impacts of cultivars, phosphorus and $\mathrm{C} \times \mathrm{P}$ on citrus plant growth i.e., root, branch, leaves and total plant dry weight were observed. Compared with LP treatment, middle level phosphorus supplement (MP) significantly increased root dry weight, branch dry weight, leaves dry weight, and total dry weight by $18.41 \%, 79.84 \%$, $91.65 \%$, and $67.72 \%$, respectively; high level phosphorus supplement (HP) remarkably improved root dry weight, branch dry weight, leaves dry weight, and total dry weight by $18.18 \%, 85.05 \%, 103.21 \%$, and $74.15 \%$, respectively. For MP treatment, higher root dry weight (73.79\% and 50.28\%), leaves dry weight (200.78\% and $154.76 \%)$, total dry weight $(166.43 \%$ and $108.77 \%$ ) in Cuningmeng and Suanju; while higher branch dry weight (190.57\%, 115.32\% and 116.16\%) was investigated in Cuningmeng, Suanju and Suncheng. However, the root dry weight in Hongju and Xiangcheng was inhibited by $34.53 \%$ and $30.63 \%$, respectively. Zhike gained lower branch, leaves and total dry weight improvement, Goutoucheng had lower branch dry weight improvement, and lower leaves dry weight and total dry weight improvement in Hongju was observed. For HP treatment, Cuningmeng and Suanju produced higher root dry weight $(85.24 \%$ and $115.08 \%)$, branch dry weight (116.60\% and 246.77\%), leaves dry weight (167.03\% and $279.21 \%$ ), and total dry weight (133.40\% and $214.10 \%)$. However, the root dry weight in Suancheng, Goutoucheng, Hongju, Xiangcheng, Zhicheng was inhibited. Zhike and Zhicheng had lower branch dry weight, leaves dry weight and total dry weight gained. 
Therefore, it can be stated that Cuningmeng and Suanju were high P efficiency cultivars but Zhike, Zhicheng or Hongju were low phosphorus efficiency cultivars (Table 1).

Table 1. Effects of phosphorus treatment on citrus plant growth.

\begin{tabular}{|c|c|c|c|c|c|}
\hline Phosphorus & Cultivar & $\begin{array}{c}\text { Root dry } \\
\text { weight (g) }\end{array}$ & $\begin{array}{c}\text { Branch dry } \\
\text { weight (g) }\end{array}$ & $\begin{array}{c}\text { Leaves dry } \\
\text { weight (g) }\end{array}$ & $\begin{array}{c}\text { Total dry } \\
\text { weight (g) }\end{array}$ \\
\hline \multirow[t]{11}{*}{ LP } & Cuningmeng & $1.31 \pm 0.14$ & $1.38 \pm 0.09$ & $2.56 \pm 0.25$ & $5.25 \pm 0.32$ \\
\hline & Suanju & $0.60 \pm 0.03$ & $0.41 \pm 0.05$ & $0.70 \pm 0.09$ & $1.71 \pm 0.15$ \\
\hline & Suancheng & $1.31 \pm 0.12$ & $1.00 \pm 0.14$ & $2.39 \pm 0.27$ & $4.70 \pm 0.47$ \\
\hline & Honglimeng & $1.53 \pm 0.10$ & $2.09 \pm 0.17$ & $2.74 \pm 0.17$ & $6.36 \pm 0.43$ \\
\hline & Zhike & $0.76 \pm 0.08$ & $0.79 \pm 0.02$ & $0.89 \pm 0.05$ & $2.44 \pm 0.13$ \\
\hline & Goutoucheng & $0.70 \pm 0.03$ & $0.86 \pm 0.07$ & $1.26 \pm 0.17$ & $2.83 \pm 0.27$ \\
\hline & Xiangyuan & $1.61 \pm 0.13$ & $2.52 \pm 0.16$ & $3.21 \pm 0.23$ & $7.34 \pm 0.48$ \\
\hline & Hongju & $1.02 \pm 0.06$ & $0.38 \pm 0.04$ & $0.88 \pm 0.07$ & $2.29 \pm 0.15$ \\
\hline & Xiangcheng & $1.07 \pm 0.06$ & $1.02 \pm 0.06$ & $1.83 \pm 0.08$ & $3.92 \pm 0.19$ \\
\hline & Zhicheng & $1.67 \pm 0.12$ & $1.34 \pm 0.06$ & $1.44 \pm 0.10$ & $4.44 \pm 0.16$ \\
\hline & Mean & $1.1587 b$ & $1.1781 b$ & $1.7902 b$ & $4.1269 b$ \\
\hline \multirow[t]{11}{*}{ MP } & Cuningmeng & $2.28 \pm 0.25$ & $4.01 \pm 0.22$ & $7.71 \pm 0.47$ & $14.00 \pm 0.89$ \\
\hline & Suanju & $0.90 \pm 0.10$ & $0.89 \pm 0.03$ & $1.78 \pm 0.16$ & $3.57 \pm 0.29$ \\
\hline & Suancheng & $1.69 \pm 0.06$ & $2.15 \pm 0.11$ & $4.44 \pm 0.22$ & $8.28 \pm 0.38$ \\
\hline & Honglimeng & $1.92 \pm 0.10$ & $3.44 \pm 0.23$ & $4.93 \pm 0.32$ & $10.29 \pm 0.46$ \\
\hline & Zhike & $0.98 \pm 0.09$ & $0.97 \pm 0.08$ & $1.14 \pm 0.13$ & $3.09 \pm 0.29$ \\
\hline & Goutoucheng & $0.82 \pm 0.13$ & $1.17 \pm 0.06$ & $2.06 \pm 0.17$ & $4.04 \pm 0.32$ \\
\hline & Xiangyuan & $1.94 \pm 0.12$ & $4.29 \pm 0.16$ & $5.93 \pm 0.09$ & $12.16 \pm 0.37$ \\
\hline & Hongju & $0.67 \pm 0.03$ & $0.71 \pm 0.06$ & $1.32 \pm 0.09$ & $2.70 \pm 0.12$ \\
\hline & Xiangcheng & $0.74 \pm 0.08$ & $1.60 \pm 0.17$ & $2.82 \pm 0.20$ & $5.16 \pm 0.42$ \\
\hline & Zhicheng & $1.78 \pm 0.14$ & $1.95 \pm 0.03$ & $2.18 \pm 0.05$ & $5.91 \pm 0.20$ \\
\hline & Mean & $1.3720 a$ & $2.1187 a$ & $3.4308 a$ & $6.9215 a$ \\
\hline \multirow[t]{11}{*}{ HP } & Cuningmeng & $2.43 \pm 0.18$ & $2.99 \pm 0.16$ & $6.85 \pm 0.61$ & $12.26 \pm 0.76$ \\
\hline & Suanju & $1.28 \pm 0.16$ & $1.43 \pm 0.10$ & $2.65 \pm 0.23$ & $5.37 \pm 0.43$ \\
\hline & Suancheng & $1.08 \pm 0.09$ & $1.46 \pm 0.21$ & $3.57 \pm 0.21$ & $6.12 \pm 0.44$ \\
\hline & Honglimeng & $1.78 \pm 0.24$ & $3.66 \pm 0.21$ & $5.72 \pm 0.23$ & $11.15 \pm 0.60$ \\
\hline & Zhike & $0.86 \pm 0.09$ & $1.01 \pm 0.05$ & $1.30 \pm 0.09$ & $3.17 \pm 0.23$ \\
\hline & Goutoucheng & $0.63 \pm 0.04$ & $1.33 \pm 0.08$ & $2.13 \pm 0.11$ & $4.09 \pm 0.20$ \\
\hline & Xiangyuan & $2.50 \pm 0.31$ & $5.16 \pm 0.39$ & $6.90 \pm 0.61$ & $14.55 \pm 1.3$ \\
\hline & Hongju & $0.82 \pm 0.10$ & $0.91 \pm 0.04$ & $1.77 \pm 0.1$ & $3.51 \pm 0.18$ \\
\hline & Xiangcheng & $1.00 \pm 0.08$ & $2.37 \pm 0.13$ & $3.82 \pm 0.19$ & $7.19 \pm 0.30$ \\
\hline & Zhicheng & $1.31 \pm 0.09$ & $1.48 \pm 0.10$ & $1.67 \pm 0.12$ & $4.45 \pm 0.28$ \\
\hline & Mean & $1.3693 a$ & $2.1801 a$ & $3.6378 a$ & $7.1872 a$ \\
\hline \multicolumn{6}{|l|}{ F value } \\
\hline & Cultivar(C) & $53.07 * *$ & $186.02 * *$ & $143.66 * *$ & $150.66 * *$ \\
\hline & Phosphorus (P) & $8.11 * *$ & $154.92 * *$ & $174.77 * *$ & $140.75^{* *}$ \\
\hline & $\mathrm{C} \times \mathrm{P}$ & $4.91 * *$ & $13.33 * *$ & $13.29 * *$ & $12.98 * *$ \\
\hline
\end{tabular}

Note: different lowercase letters are used to indicate values that are significantly different at $p<0.05$ among P treatments. 


\section{$N$ content and $N$ accumulation}

Significant effects of cultivars, phosphorus and $\mathrm{C} \times \mathrm{P}$ on citrus plant $\mathrm{N}$ content and accumulation in different plant part were observed, except for the $\mathrm{C} \times \mathrm{P}$ effect on $\mathrm{N}$ content in branch. MP treatment significantly increased $\mathrm{N}$ content in root, branch, leaves, $\mathrm{N}$ accumulation in root, $\mathrm{N}$ accumulation in branch, $\mathrm{N}$ accumulation in leaves, and total $\mathrm{N}$ accumulation by $34.52 \%, 24.58 \%, 24.43 \%, 56.24 \%, 112.35 \%, 124.73 \%$, and $103.25 \%$, respectively. HP treatment significantly increased the $\mathrm{N}$ content in root, branch, leaves, $\mathrm{N}$ accumulation in root, $\mathrm{N}$ accumulation in branch, $\mathrm{N}$ accumulation in leaves, and total $\mathrm{N}$ accumulation by $31.82 \%, 29.35 \%, 25.87 \%, 52.09 \%, 126.56 \%$, $142.72 \%$, and $114.33 \%$, respectively. Highest $\mathrm{N}$ content increment were observed in Goutoucheng for MP and HP treatment, while lowest increment in N content in branch and leaves were investigated in Cuningmeng for MP and HP. The highest increment in $\mathrm{N}$ content in branch and leaves were found in Quenching for MP and in Goutoucheng for HP. For the accumulation of $\mathrm{N}$ in citrus plant, Hongju and Xiangcheng had lower $\mathrm{N}$ accumulation in root for MP treatment, the highest $\mathrm{N}$ accumulation in branch was observed in Suancheng but the lowest $\mathrm{N}$ accumulation in branch was investigated in Zhike for MP. Higher $\mathrm{N}$ accumulation in leaves and total $\mathrm{N}$ accumulation were detected in Suancheng and lower $\mathrm{N}$ accumulation in leaves and total $\mathrm{N}$ accumulation were found in Zhike for MP. Suancheng had the highest N accumulation in plant while Zhicheng had the highest $\mathrm{N}$ accumulation in plant for HP (Table 2).

\section{$P$ content and $P$ accumulation}

Cultivars, phosphorus and $\mathrm{C} \times \mathrm{P}$ significantly affected $\mathrm{P}$ accumulation in citrus plants. MP treatment significantly increased $\mathrm{P}$ content in root, branch, and leaves. $\mathrm{P}$ accumulation in root, $\mathrm{P}$ accumulation in branch, $\mathrm{P}$ accumulation in leaves, and total $\mathrm{P}$ accumulation increased by $182.05 \%, 189.96 \%, 193.30 \%, 231.51 \%, 420.40 \%, 474.22 \%$, and $388.03 \%$, respectively. HP treatment dramatically increased $\mathrm{P}$ content in root, $\mathrm{P}$ content in branch, $\mathrm{P}$ content in leaves, $\mathrm{P}$ accumulation in root, $\mathrm{P}$ accumulation in branch, $\mathrm{P}$ accumulation in leaves, and total $\mathrm{P}$ accumulation by $211.06 \%, 197.08 \%$, 205.49\%, 257.99\%, 455.24\%, 547.75\%, and 437.76\%, respectively. For MP treatment, the increment in $\mathrm{P}$ content in root ranged from $129.46 \%$ to $298.85 \%$, P content in branch ranged from $72.85 \%$ to $251.95 \%$, $\mathrm{P}$ content in leaves ranged from $73.82 \%$ to $272.43 \%$ and $\mathrm{P}$ accumulation in root ranged from $73.08 \%$ to $586.10 \%, \mathrm{P}$ accumulation in branch ranged from $113.59 \%$ to $847.09 \%, \mathrm{P}$ accumulation in leaves ranged from $124.77 \%$ to $901.49 \%$, and total $\mathrm{P}$ accumulation ranged from $145.38 \%$ to $812.48 \%$. The data showed that Cuningmeng had higher increment in P content and accumulation, but that of Zhike was lower. For HP treatment, the increment in P content in root ranged from $134.58 \%$ to $347.99 \%$, $\mathrm{P}$ content in branch ranged from $83.32 \%$ to $291.78 \%, \mathrm{P}$ content in leaves ranged from $80.36 \%$ to $304.84 \%$ and $\mathrm{P}$ accumulation in root ranged from $107.59 \%$ to $725.85 \%, \mathrm{P}$ accumulation in branch ranged from $134.27 \%$ to $834.43 \%, \mathrm{P}$ accumulation in leaves ranged from $164.64 \%$ to $920.37 \%$, and total $\mathrm{P}$ accumulation ranged from $162.06 \%$ to $961.33 \%$. Among the cultivars, Cuningmeng showed higher $\mathrm{P}$ content and accumulation, but lower $\mathrm{P}$ content and accumulation was observed for Zhike (Table 3). 
Table 2. Effects of phosphorus treatment on $N$ content and $N$ accumulation in citrus plants.

\begin{tabular}{|c|c|c|c|c|c|c|c|c|}
\hline Phosphorus & Cultivar & $\begin{array}{c}\mathrm{N} \text { content in } \\
\operatorname{root} \\
\left(\mathrm{mg} \mathrm{g}^{-1}\right)\end{array}$ & $\begin{array}{l}\text { N content in } \\
\text { branch } \\
\left(\mathrm{mg} \mathrm{g}^{-1}\right)\end{array}$ & $\begin{array}{c}\mathbf{N} \text { content in } \\
\text { leaves } \\
\left(\mathrm{mg} \mathrm{g}^{-1}\right)\end{array}$ & $\begin{array}{c}\mathrm{N} \\
\text { accumulation } \\
\text { in root }(\mathbf{m g})\end{array}$ & $\begin{array}{c}\mathbf{N} \\
\text { accumulation } \\
\text { in branch }(\mathbf{m g})\end{array}$ & $\begin{array}{c}\mathbf{N} \\
\text { accumulation in } \\
\text { leaves }(\mathbf{m g})\end{array}$ & $\begin{array}{c}\text { Total } \mathbf{N} \\
\text { accumulation } \\
(\mathbf{m g})\end{array}$ \\
\hline \multirow[t]{11}{*}{ LP } & Cuningmeng & $19.73 \pm 2.13$ & $8.60 \pm 0.52$ & $25.47 \pm 1.54$ & $26.00 \pm 4.21$ & $11.95 \pm 1.47$ & $66.07 \pm 10.45$ & $104.02 \pm 8.82$ \\
\hline & Suanju & $16.17 \pm 0.81$ & $11.42 \pm 0.85$ & $24.48 \pm 2.33$ & $9.68 \pm 0.94$ & $4.65 \pm 0.26$ & $16.74 \pm 1.13$ & $31.06 \pm 1.32$ \\
\hline & Suancheng & $20.37 \pm 0.41$ & $10.02 \pm 0.32$ & $18.39 \pm 1.07$ & $26.67 \pm 1.99$ & $9.98 \pm 1.41$ & $43.30 \pm 2.57$ & $79.94 \pm 5.27$ \\
\hline & Honglimeng & $18.97 \pm 0.54$ & $8.63 \pm 0.07$ & $22.96 \pm 1.16$ & $29.12 \pm 2.70$ & $18.02 \pm 1.53$ & $62.60 \pm 1.89$ & $109.74 \pm 4.86$ \\
\hline & Zhike & $18.27 \pm 0.83$ & $12.38 \pm 1.18$ & $26.48 \pm 2.27$ & $13.75 \pm 0.87$ & $9.73 \pm 0.70$ & $23.63 \pm 2.77$ & $47.11 \pm 4.16$ \\
\hline & Goutoucheng & $23.13 \pm 2.33$ & $11.10 \pm 0.99$ & $24.93 \pm 1.48$ & $16.40 \pm 2.37$ & $9.43 \pm 0.42$ & $31.08 \pm 2.41$ & $56.91 \pm 4.58$ \\
\hline & Xiangyuan & $16.67 \pm 0.82$ & $9.57 \pm 1.01$ & $26.46 \pm 2.35$ & $27.00 \pm 3.20$ & $24.39 \pm 4.15$ & $85.97 \pm 14.12$ & $137.36 \pm 21.14$ \\
\hline & Hongju & $18.97 \pm 1.08$ & $11.60 \pm 0.71$ & $24.72 \pm 0.86$ & $19.52 \pm 2.22$ & $4.47 \pm 0.70$ & $21.95 \pm 2.42$ & $45.94 \pm 5.11$ \\
\hline & Xiangcheng & $28.80 \pm 1.27$ & $11.24 \pm 0.23$ & $24.45 \pm 0.35$ & $30.87 \pm 3.09$ & $11.49 \pm 0.83$ & $44.75 \pm 2.39$ & $87.12 \pm 6.25$ \\
\hline & Zhicheng & $15.27 \pm 0.69$ & $13.36 \pm 0.98$ & $30.15 \pm 2.32$ & $25.56 \pm 2.49$ & $17.97 \pm 2.04$ & $43.83 \pm 6.10$ & $87.35 \pm 8.58$ \\
\hline & Mean & $19.633 b$ & $10.793 b$ & $24.850 \mathrm{~b}$ & $22.457 b$ & $12.208 b$ & $43.99 c$ & $78.66 b$ \\
\hline \multirow[t]{11}{*}{ MP } & Cuningmeng & $26.4 \pm 1.72$ & $8.31 \pm 0.53$ & $23.53 \pm 0.41$ & $59.68 \pm 5.66$ & $33.47 \pm 3.80$ & $181.16 \pm 8.26$ & $274.31 \pm 17.69$ \\
\hline & Suanju & $20.70 \pm 0.93$ & $13.76 \pm 1.03$ & $29.58 \pm 1.45$ & $18.60 \pm 2.33$ & $12.22 \pm 0.88$ & $52.88 \pm 6.00$ & $83.71 \pm 9.06$ \\
\hline & Suancheng & $29.73 \pm 1.53$ & $14.25 \pm 0.33$ & $26.45 \pm 0.95$ & $50.21 \pm 2.77$ & $30.75 \pm 2.14$ & $117.49 \pm 7.75$ & $198.45 \pm 11.00$ \\
\hline & Honglimeng & $21.53 \pm 1.77$ & $9.77 \pm 0.75$ & $25.49 \pm 2.07$ & $41.37 \pm 4.20$ & $33.51 \pm 2.59$ & $125.79 \pm 13.29$ & $200.68 \pm 11.88$ \\
\hline & Zhike & $22.40 \pm 1.07$ & $14.32 \pm 1.60$ & $34.42 \pm 1.53$ & $21.87 \pm 1.34$ & $13.89 \pm 1.95$ & $39.51 \pm 5.70$ & $75.28 \pm 8.42$ \\
\hline & Goutoucheng & $34.70 \pm 0.64$ & $14.61 \pm 0.65$ & $35.10 \pm 1.32$ & $28.52 \pm 4.70$ & $17.01 \pm 0.63$ & $71.70 \pm 3.48$ & $117.23 \pm 6.61$ \\
\hline & Xiangyuan & $25.70 \pm 0.31$ & $11.72 \pm 0.66$ & $30.76 \pm 1.06$ & $49.81 \pm 2.69$ & $50.45 \pm 4.38$ & $182.53 \pm 9.14$ & $282.78 \pm 15.48$ \\
\hline & Hongju & $25.87 \pm 0.93$ & $15.96 \pm 0.59$ & $33.70 \pm 1.22$ & $17.36 \pm 1.14$ & $11.30 \pm 0.85$ & $44.36 \pm 1.73$ & $73.02 \pm 1.54$ \\
\hline & Xiangcheng & $36.70 \pm 0.31$ & $14.75 \pm 0.58$ & $32.14 \pm 1.15$ & $27.16 \pm 2.99$ & $23.42 \pm 1.97$ & $90.30 \pm 4.18$ & $140.88 \pm 8.97$ \\
\hline & Zhicheng & $20.37 \pm 0.13$ & $17.02 \pm 0.21$ & $38.04 \pm 0.59$ & $36.29 \pm 2.56$ & $33.20 \pm 0.36$ & $82.89 \pm 1.19$ & $152.37 \pm 4.07$ \\
\hline & Mean & $25.880 a$ & $13.446 a$ & $30.921 a$ & $35.087 a$ & $25.923 a$ & $98.86 b$ & $159.87 a$ \\
\hline \multirow[t]{2}{*}{ HP } & Cuningmeng & $24.07 \pm 2.29$ & $9.30 \pm 1.02$ & $25.57 \pm 2.26$ & $58.97 \pm 9.42$ & $27.56 \pm 2.55$ & $172.35 \pm 1.08$ & $258.87 \pm 9.40$ \\
\hline & Suanju & $22.67 \pm 1.90$ & $15.20 \pm 0.67$ & $31.24 \pm 1.07$ & $29.69 \pm 6.30$ & $21.68 \pm 0.83$ & $82.76 \pm 6.75$ & $134.13 \pm 10.58$ \\
\hline
\end{tabular}




\begin{tabular}{|c|c|c|c|c|c|c|c|c|}
\hline & Suancheng & $28.17 \pm 2.87$ & $14.57 \pm 1.00$ & $29.01 \pm 1.53$ & $30.76 \pm 4.95$ & $20.93 \pm 1.43$ & $103.02 \pm 0.68$ & $154.72 \pm 4.52$ \\
\hline & Honglimeng & $22.73 \pm 1.63$ & $11.57 \pm 1.69$ & $27.68 \pm 1.16$ & $40.91 \pm 7.40$ & $41.73 \pm 4.22$ & $158.32 \pm 9.88$ & $240.97 \pm 19.34$ \\
\hline & Zhike & $23.63 \pm 0.50$ & $15.13 \pm 1.06$ & $31.40 \pm 2.18$ & $20.29 \pm 2.11$ & $15.17 \pm 0.51$ & $40.47 \pm 0.73$ & $75.93 \pm 2.17$ \\
\hline & Goutoucheng & $37.53 \pm 1.34$ & $16.57 \pm 0.47$ & $38.14 \pm 0.42$ & $23.56 \pm 0.79$ & $21.96 \pm 0.94$ & $81.29 \pm 3.50$ & $126.80 \pm 4.01$ \\
\hline & Xiangyuan & $23.40 \pm 2.29$ & $11.12 \pm 1.64$ & $28.22 \pm 3.04$ & $60.02 \pm 12.87$ & $56.41 \pm 5.65$ & $192.00 \pm 13.55$ & $308.43 \pm 19.53$ \\
\hline & Hongju & $25.47 \pm 0.60$ & $15.97 \pm 0.47$ & $33.56 \pm 0.48$ & $20.90 \pm 2.71$ & $14.58 \pm 0.49$ & $59.47 \pm 2.96$ & $94.96 \pm 5.62$ \\
\hline & Xiangcheng & $32.73 \pm 0.99$ & $13.07 \pm 0.88$ & $29.96 \pm 1.05$ & $32.59 \pm 1.52$ & $31.19 \pm 3.82$ & $114.77 \pm 9.86$ & $178.54 \pm 13.53$ \\
\hline & Zhicheng & $18.40 \pm 1.85$ & $17.10 \pm 0.20$ & $37.99 \pm 0.78$ & $23.86 \pm 1.90$ & $25.36 \pm 1.78$ & $63.31 \pm 4.96$ & $112.54 \pm 8.53$ \\
\hline & Mean & $26.410 a$ & $13.960 a$ & $31.279 a$ & $34.155 a$ & $27.657 a$ & $106.78 a$ & $168.59 a$ \\
\hline$F$ value & & & & & & & & \\
\hline & Cultivar(C) & $36.05^{* *}$ & $29.16^{* *}$ & $15.94 * *$ & $22.81 * *$ & $119.82 * *$ & $114.83 * *$ & $98.11 * *$ \\
\hline & Phosphorus (P) & $70.17 * *$ & $33.05^{* *}$ & $56.86^{* * *}$ & $23.16^{* * *}$ & $109.86^{* *}$ & $276.30 * *$ & $251.27 * *$ \\
\hline & $\mathrm{C} \times \mathrm{P}$ & $1.91 *$ & $1.10 \mathrm{~ns}$ & $2.46 * *$ & $3.01 * *$ & $4.33 * *$ & $9.62 * *$ & $10.26 * *$ \\
\hline
\end{tabular}

Note: different lowercase letters are used to indicate values that are significantly different at $p<0.05$ among P treatments.

Table 3. Effects of phosphorus treatment on $P$ content and $P$ accumulation in citrus plants.

\begin{tabular}{|c|c|c|c|c|c|c|c|c|}
\hline Phosphorus & Cultivar & $\begin{array}{l}P \text { content in } \\
\operatorname{root}\left(\mathrm{mg} \mathrm{g}^{-1}\right)\end{array}$ & $\begin{array}{c}P \text { content in } \\
\operatorname{branch}\left(\mathrm{mg} \mathrm{g}^{-1}\right)\end{array}$ & $\begin{array}{l}P \text { content in } \\
\text { leaves }\left(\mathrm{mg} \mathrm{g}^{-1}\right)\end{array}$ & $\begin{array}{c}\mathbf{P} \\
\text { accumulation } \\
\text { in root (mg) } \\
\end{array}$ & $\begin{array}{c}\mathbf{P} \\
\text { accumulation in } \\
\text { branch (mg) }\end{array}$ & $\begin{array}{c}\mathbf{P} \\
\text { accumulation } \\
\text { in leaves (mg) }\end{array}$ & $\begin{array}{c}\text { Total } \mathbf{P} \\
\text { accumulation } \\
(\mathbf{m g})\end{array}$ \\
\hline \multirow[t]{9}{*}{ LP } & Cuningmeng & $0.64 \pm 0.03$ & $0.54 \pm 0.02$ & $0.72 \pm 0.01$ & $0.84 \pm 0.13$ & $0.75 \pm 0.07$ & $1.85 \pm 0.19$ & $3.45 \pm 0.25$ \\
\hline & Suanju & $1.22 \pm 0.08$ & $1.02 \pm 0.11$ & $1.06 \pm 0.05$ & $0.72 \pm 0.02$ & $0.43 \pm 0.09$ & $0.74 \pm 0.11$ & $1.90 \pm 0.19$ \\
\hline & Suancheng & $0.84 \pm 0.06$ & $0.83 \pm 0.09$ & $0.72 \pm 0.06$ & $1.10 \pm 0.12$ & $0.80 \pm 0.05$ & $1.73 \pm 0.28$ & $3.63 \pm 0.35$ \\
\hline & Honglimeng & $0.82 \pm 0.06$ & $0.73 \pm 0.01$ & $0.88 \pm 0.07$ & $1.26 \pm 0.15$ & $1.52 \pm 0.13$ & $2.38 \pm 0.09$ & $5.16 \pm 0.23$ \\
\hline & Zhike & $1.08 \pm 0.11$ & $1.19 \pm 0.08$ & $1.32 \pm 0.13$ & $0.82 \pm 0.12$ & $0.94 \pm 0.04$ & $1.17 \pm 0.11$ & $2.93 \pm 0.16$ \\
\hline & Goutoucheng & $0.99 \pm 0.00$ & $0.83 \pm 0.02$ & $0.81 \pm 0.05$ & $0.70 \pm 0.03$ & $0.72 \pm 0.08$ & $1.03 \pm 0.18$ & $2.45 \pm 0.28$ \\
\hline & Xiangyuan & $0.91 \pm 0.06$ & $0.51 \pm 0.04$ & $1.01 \pm 0.04$ & $1.48 \pm 0.22$ & $1.28 \pm 0.17$ & $3.25 \pm 0.38$ & $6.01 \pm 0.73$ \\
\hline & Hongju & $1.17 \pm 0.10$ & $0.89 \pm 0.02$ & $0.90 \pm 0.06$ & $1.21 \pm 0.16$ & $0.34 \pm 0.04$ & $0.80 \pm 0.11$ & $2.35 \pm 0.30$ \\
\hline & Xiangcheng & $0.87 \pm 0.04$ & $0.84 \pm 0.02$ & $0.79 \pm 0.02$ & $0.92 \pm 0.01$ & $0.85 \pm 0.05$ & $1.45 \pm 0.08$ & $3.23 \pm 0.14$ \\
\hline
\end{tabular}




\begin{tabular}{|c|c|c|c|c|c|c|c|c|}
\hline \multirow{4}{*}{ MP } & Zhicheng & $0.96 \pm 0.09$ & $0.96 \pm 0.09$ & $1.09 \pm 0.09$ & $1.61 \pm 0.22$ & $1.30 \pm 0.17$ & $1.58 \pm 0.23$ & $4.49 \pm 0.25$ \\
\hline & Mean & $0.9497 c$ & $0.8337 b$ & $0.9307 c$ & $1.0657 b$ & $0.8936 c$ & $1.600 c$ & $3.559 c$ \\
\hline & Cuningmeng & $2.54 \pm 0.17$ & $1.77 \pm 0.07$ & $2.41 \pm 0.08$ & $5.79 \pm 0.75$ & $7.09 \pm 0.45$ & $18.58 \pm 0.90$ & $31.45 \pm 1.93$ \\
\hline & Suanju & $3.03 \pm 0.10$ & $3.04 \pm 0.19$ & $2.98 \pm 0.12$ & $2.72 \pm 0.34$ & $2.72 \pm 0.27$ & $5.36 \pm 0.73$ & $10.80 \pm 1.33$ \\
\hline \multirow{19}{*}{ HP } & Suancheng & $2.63 \pm 0.07$ & $2.69 \pm 0.05$ & $2.38 \pm 0.05$ & $4.45 \pm 0.26$ & $5.81 \pm 0.34$ & $10.60 \pm 0.72$ & $20.85 \pm 1.30$ \\
\hline & Honglimeng & $2.39 \pm 0.10$ & $2.41 \pm 0.13$ & $3.13 \pm 0.21$ & $4.58 \pm 0.26$ & $8.25 \pm 0.38$ & $15.27 \pm 0.25$ & $28.10 \pm 0.35$ \\
\hline & Zhike & $2.58 \pm 0.06$ & $2.07 \pm 0.01$ & $2.30 \pm 0.07$ & $2.55 \pm 0.28$ & $2.01 \pm 0.18$ & $2.63 \pm 0.35$ & $7.19 \pm 0.80$ \\
\hline & Goutoucheng & $3.25 \pm 0.05$ & $2.75 \pm 0.04$ & $2.89 \pm 0.05$ & $2.67 \pm 0.44$ & $3.21 \pm 0.15$ & $5.91 \pm 0.39$ & $11.79 \pm 0.87$ \\
\hline & Xiangyuan & $2.39 \pm 0.23$ & $1.61 \pm 0.08$ & $2.80 \pm 0.14$ & $4.64 \pm 0.51$ & $6.91 \pm 0.59$ & $16.61 \pm 1.08$ & $28.17 \pm 1.95$ \\
\hline & Hongju & $3.12 \pm 0.04$ & $3.12 \pm 0.19$ & $3.36 \pm 0.17$ & $2.09 \pm 0.06$ & $2.19 \pm 0.06$ & $4.41 \pm 0.11$ & $8.69 \pm 0.06$ \\
\hline & Xiangcheng & $2.65 \pm 0.07$ & $2.51 \pm 0.05$ & $2.40 \pm 0.05$ & $1.97 \pm 0.26$ & $4.00 \pm 0.39$ & $6.78 \pm 0.52$ & $12.75 \pm 1.10$ \\
\hline & Zhicheng & $2.2 \pm 0.14$ & $2.21 \pm 0.03$ & $2.62 \pm 0.07$ & $3.88 \pm 0.03$ & $4.32 \pm 0.05$ & $5.71 \pm 0.16$ & $13.91 \pm 0.22$ \\
\hline & Mean & $2.6773 b$ & $2.4173 a$ & $2.7263 b$ & $3.5331 a$ & $4.6501 b$ & $9.187 b$ & $17.370 b$ \\
\hline & Cuningmeng & $2.86 \pm 0.17$ & $2.12 \pm 0.15$ & $2.92 \pm 0.13$ & $6.97 \pm 0.85$ & $6.29 \pm 0.29$ & $19.88 \pm 0.96$ & $33.14 \pm 1.11$ \\
\hline & Suanju & $3.63 \pm 0.12$ & $2.83 \pm 0.05$ & $2.86 \pm 0.04$ & $4.64 \pm 0.56$ & $4.05 \pm 0.20$ & $7.56 \pm 0.57$ & $16.26 \pm 1.22$ \\
\hline & Suancheng & $3.41 \pm 0.06$ & $2.96 \pm 0.1$ & $2.59 \pm 0.11$ & $3.70 \pm 0.36$ & $4.31 \pm 0.53$ & $9.23 \pm 0.33$ & $17.24 \pm 1.08$ \\
\hline & Honglimeng & $2.08 \pm 0.09$ & $2.32 \pm 0.18$ & $3.10 \pm 0.22$ & $3.66 \pm 0.37$ & $8.55 \pm 1.11$ & $17.81 \pm 2.02$ & $30.02 \pm 3.34$ \\
\hline & Zhike & $2.75 \pm 0.10$ & $2.19 \pm 0.08$ & $2.38 \pm 0.06$ & $2.38 \pm 0.33$ & $2.20 \pm 0.06$ & $3.09 \pm 0.19$ & $7.67 \pm 0.54$ \\
\hline & Goutoucheng & $4.05 \pm 0.07$ & $2.74 \pm 0.02$ & $2.99 \pm 0.04$ & $2.56 \pm 0.19$ & $3.63 \pm 0.2$ & $6.38 \pm 0.40$ & $12.57 \pm 0.62$ \\
\hline & Xiangyuan & $2.13 \pm 0.18$ & $1.67 \pm 0.15$ & $2.95 \pm 0.20$ & $5.44 \pm 1.10$ & $8.50 \pm 0.37$ & $20.11 \pm 0.59$ & $34.05 \pm 1.53$ \\
\hline & Hongju & $3.32 \pm 0.10$ & $3.19 \pm 0.07$ & $3.50 \pm 0.05$ & $2.73 \pm 0.38$ & $2.92 \pm 0.18$ & $6.21 \pm 0.40$ & $11.86 \pm 0.68$ \\
\hline & Xiangcheng & $2.74 \pm 0.02$ & $2.35 \pm 0.07$ & $2.23 \pm 0.04$ & $2.74 \pm 0.19$ & $5.58 \pm 0.42$ & $8.52 \pm 0.57$ & $16.83 \pm 0.95$ \\
\hline & Zhicheng & $2.56 \pm 0.14$ & $2.4 \pm 0.16$ & $2.89 \pm 0.16$ & $3.34 \pm 0.2$ & $3.58 \pm 0.43$ & $4.83 \pm 0.55$ & $11.75 \pm 0.88$ \\
\hline \multirow{4}{*}{ F value } & Mean & $2.9527 a$ & $2.4760 a$ & $2.8393 a$ & $3.8152 a$ & $4.9614 a$ & $10.363 a$ & $19.140 a$ \\
\hline & Cultivar(C) & $43.04^{* *}$ & $31.11^{* *}$ & $16.32^{* * *}$ & $16.41^{* *}$ & $45.48^{* * *}$ & $119.62 * *$ & $85.80^{* *}$ \\
\hline & Phosphorus (P) & $955.26 * *$ & $1072.93 * *$ & $964.39^{* *}$ & $147.22 * *$ & $514.50^{* *}$ & $668.34 * *$ & $667.66^{* *}$ \\
\hline & $\mathrm{C} \times \mathrm{P}$ & $7.82 * *$ & $8.18^{* *}$ & $5.61 * *$ & $4.93 * *$ & $15.35^{* * *}$ & $30.53 * *$ & 23.49 ** \\
\hline
\end{tabular}

Note: different lowercase letters are used to indicate values that are significantly different at $p<0.05$ among P treatments. 


\section{K content and K accumulation}

The cultivars, phosphorus and their interaction $\mathrm{C} \times \mathrm{P}$ significantly affected the $\mathrm{K}$ content and accumulation in citrus plant. MP treatment significantly increased $\mathrm{K}$ content in root, $\mathrm{K}$ content in branch, $\mathrm{K}$ content in leaves, $\mathrm{K}$ accumulation in root, $\mathrm{K}$ accumulation in branch, $\mathrm{K}$ accumulation in leaves, and total $\mathrm{K}$ accumulation by $51.99 \%, 21.46 \%, 21.27 \%, 87.18 \%, 122.44 \%, 138.65 \%$, and $124.73 \%$, respectively. HP treatment dramatically increased $\mathrm{K}$ content in root, $\mathrm{K}$ content in branch, $\mathrm{K}$ content in leaves, $\mathrm{K}$ accumulation in root, $\mathrm{K}$ accumulation in branch, $\mathrm{K}$ accumulation in leaves, and total $\mathrm{K}$ accumulation by $41.09 \%, 17.01 \%, 16.64 \%, 69.81 \%, 120.57 \%, 142.22 \%$, and $122.91 \%$, respectively. For MP treatment, Xiangyuan had the highest increment in $\mathrm{K}$ content in root, $\mathrm{K}$ content in branch, $\mathrm{K}$ content in leaves and $\mathrm{K}$ accumulation in root; while Cuningmeng had the highest increment in $\mathrm{K}$ accumulation in branch, $\mathrm{K}$ accumulation in leaves, and total $\mathrm{K}$ accumulation. The lowest increment in $\mathrm{K}$ content in branch, $\mathrm{K}$ content in leaves, $\mathrm{K}$ accumulation in branch, $\mathrm{K}$ accumulation in leaves, and total $\mathrm{K}$ accumulation was observed in Zhike for MP treatment. The lowest increment in $\mathrm{K}$ content in root and $\mathrm{K}$ accumulation in root was investigated in Suanju and Hongju for MP, respectively. For HP treatment, Cuningmeng had the highest increment in K content in root and $\mathrm{K}$ accumulation in root when compared to LP. Suanju decreased $\mathrm{K}$ content in branch and $\mathrm{K}$ content in leaves but had the highest increment in $\mathrm{K}$ accumulation in branch, $\mathrm{K}$ accumulation in leaves, and total $\mathrm{K}$ accumulation for $\mathrm{HP}$ treatment. Hongju had the highest increment in $\mathrm{K}$ content in branch and $\mathrm{K}$ content in leaves. Zhicheng showed lowest highest increment in $\mathrm{K}$ accumulation (Table 4).

\section{Mg content and Mg accumulation}

The cultivars, phosphorus and $\mathrm{C} \times \mathrm{P}$ on $\mathrm{Mg}$ content and $\mathrm{Mg}$ accumulation in different plant part were observed, except for the $\mathrm{P}$ treatment effect on $\mathrm{Mg}$ content in leaves and $\mathrm{C} \times \mathrm{P}$ effect on $\mathrm{Mg}$ content in branch and leaves. MP treatment significantly increased $\mathrm{Mg}$ content in root, $\mathrm{Mg}$ accumulation in root, $\mathrm{Mg}$ accumulation in branch, $\mathrm{Mg}$ accumulation in leaves, and total $\mathrm{Mg}$ accumulation by $26.58 \%, 52.06 \%, 81.35 \%$, $95.35 \%$ and $84.81 \%$, respectively. HP treatment dramatically increased $\mathrm{Mg}$ content in root, $\mathrm{Mg}$ content in branch, $\mathrm{Mg}$ accumulation in root, $\mathrm{Mg}$ accumulation in branch, $\mathrm{Mg}$ accumulation in leaves, and total $\mathrm{Mg}$ accumulation by $27.88 \%, 7.65 \%, 52.30 \%$, $101.68 \%, 111.60 \%$ and $99.33 \%$, respectively. MP treatment increased $\mathrm{Mg}$ content in root in range of $7.03 \sim 76.54 \%$. However, Zhike and Hongju decreased Mg content in root for HP treatment was detected. There observed decrease in $\mathrm{Mg}$ content in branch and leaves for Cuningmeng, Suanju, Zhike, Xiangcheng for MP treatment. Mg content in branch and leaves in Suanju, Goutoucheng and xiangcheng was decreased for MP treatment, and decrement in $\mathrm{Mg}$ content in branch for Honglimeng and $\mathrm{Mg}$ content in leaves for Zhike was investigated. Decrement in $\mathrm{Mg}$ accumulation in root in Hongju was observed for MP and HP treatment and in Xiangcheng for MP treatment (Table 5). 
Table 4. Effects of phosphorus treatment on $K$ content and $K$ accumulation in citrus plants.

\begin{tabular}{|c|c|c|c|c|c|c|c|c|}
\hline Phosphorus & Cultivar & $\begin{array}{c}\mathrm{K} \text { content in } \\
\text { root } \\
\left(\mathrm{mg} \mathrm{g}^{-1}\right)\end{array}$ & $\begin{array}{c}\mathrm{K} \text { content in } \\
\text { branch } \\
\left(\mathrm{mg} \mathrm{g}^{-1}\right)\end{array}$ & $\begin{array}{c}\mathrm{K} \text { content in } \\
\text { leaves } \\
\left(\mathrm{mg} \mathrm{g}^{-1}\right)\end{array}$ & $\begin{array}{c}\mathbf{K} \\
\text { accumulation } \\
\text { in root } \\
(\mathbf{m g})\end{array}$ & $\begin{array}{c}\mathbf{K} \\
\text { accumulation } \\
\text { in branch } \\
(\mathrm{mg})\end{array}$ & $\begin{array}{c}\mathbf{K} \\
\text { accumulation } \\
\text { in leaves } \\
(\mathrm{mg})\end{array}$ & $\begin{array}{c}\text { Total K } \\
\text { accumulation } \\
(\mathbf{m g})\end{array}$ \\
\hline \multirow[t]{11}{*}{ LP } & Cuningmeng & $8.17 \pm 0.11$ & $10.33 \pm 0.34$ & $17.75 \pm 1.21$ & $10.70 \pm 1.14$ & $14.28 \pm 1.24$ & $46.02 \pm 7.17$ & $71.00 \pm 8.10$ \\
\hline & Suanju & $14.40 \pm 1.07$ & $14.05 \pm 0.35$ & $21.23 \pm 0.80$ & $8.52 \pm 0.15$ & $5.80 \pm 0.65$ & $14.75 \pm 1.60$ & $29.07 \pm 1.76$ \\
\hline & Suancheng & $11.39 \pm 0.86$ & $11.12 \pm 0.31$ & $17.43 \pm 0.59$ & $14.82 \pm 1.08$ & $11.04 \pm 1.38$ & $41.60 \pm 5.07$ & $67.46 \pm 6.67$ \\
\hline & Honglimeng & $7.52 \pm 0.09$ & $10.74 \pm 0.07$ & $21.83 \pm 0.99$ & $11.49 \pm 0.66$ & $22.4 \pm 1.81$ & $59.53 \pm 1.61$ & $93.42 \pm 3.58$ \\
\hline & Zhike & $14.43 \pm 0.68$ & $8.89 \pm 0.64$ & $18.30 \pm 1.73$ & $11.05 \pm 1.60$ & $7.00 \pm 0.35$ & $16.26 \pm 1.73$ & $34.32 \pm 3.28$ \\
\hline & Goutoucheng & $10.58 \pm 0.47$ & $11.89 \pm 0.51$ & $20.37 \pm 0.56$ & $7.46 \pm 0.58$ & $10.18 \pm 0.57$ & $25.60 \pm 2.75$ & $43.25 \pm 3.81$ \\
\hline & Xiangyuan & $7.46 \pm 0.60$ & $11.82 \pm 1.17$ & $17.74 \pm 1.88$ & $12.05 \pm 1.56$ & $30.09 \pm 4.78$ & $57.77 \pm 10.32$ & $99.91 \pm 16.5$ \\
\hline & Hongju & $10.20 \pm 0.21$ & $10.96 \pm 0.73$ & $18.37 \pm 0.81$ & $10.43 \pm 0.55$ & $4.24 \pm 0.72$ & $16.33 \pm 1.98$ & $31.01 \pm 3.09$ \\
\hline & Xiangcheng & $9.15 \pm 0.21$ & $13.50 \pm 0.35$ & $15.44 \pm 0.61$ & $9.78 \pm 0.76$ & $13.78 \pm 0.85$ & $28.31 \pm 2.20$ & $51.87 \pm 3.72$ \\
\hline & Zhicheng & $11.01 \pm 1.2$ & $13.64 \pm 0.27$ & $18.32 \pm 0.49$ & $18.63 \pm 3.08$ & $18.21 \pm 0.57$ & $26.34 \pm 1.91$ & $63.18 \pm 3.45$ \\
\hline & Mean & $10.431 c$ & $11.694 b$ & $18.680 \mathrm{~b}$ & $11.493 b$ & $13.703 b$ & $33.252 b$ & $58.45 b$ \\
\hline \multirow[t]{11}{*}{ MP } & Cuningmeng & $12.57 \pm 1.29$ & $12.90 \pm 0.30$ & $21.32 \pm 0.52$ & $28.92 \pm 5.4$ & $51.70 \pm 3.26$ & $164.16 \pm 8.36$ & $244.78 \pm 16.95$ \\
\hline & Suanju & $17.33 \pm 0.3$ & $15.02 \pm 0.53$ & $23.67 \pm 0.91$ & $15.50 \pm 1.46$ & $13.40 \pm 0.96$ & $42.25 \pm 4.52$ & $71.16 \pm 6.84$ \\
\hline & Suancheng & $18.30 \pm 1.11$ & $14.75 \pm 0.32$ & $23.16 \pm 0.62$ & $31.02 \pm 2.75$ & $31.86 \pm 2.25$ & $103.00 \pm 7.49$ & $165.87 \pm 12.21$ \\
\hline & Honglimeng & $13.70 \pm 0.40$ & $13.35 \pm 1.25$ & $26.70 \pm 1.61$ & $26.34 \pm 1.76$ & $45.48 \pm 2.44$ & $130.55 \pm 0.29$ & $202.37 \pm 3.42$ \\
\hline & Zhike & $18.87 \pm 0.64$ & $9.05 \pm 0.47$ & $17.54 \pm 0.48$ & $18.48 \pm 1.34$ & $8.86 \pm 1.13$ & $20.08 \pm 2.65$ & $47.41 \pm 5.10$ \\
\hline & Goutoucheng & $13.27 \pm 0.39$ & $14.50 \pm 0.59$ & $23.96 \pm 1.05$ & $10.95 \pm 1.93$ & $16.93 \pm 1.03$ & $48.98 \pm 3.01$ & $76.85 \pm 5.58$ \\
\hline & Xiangyuan & $19.11 \pm 0.44$ & $16.81 \pm 0.50$ & $26.24 \pm 0.86$ & $36.99 \pm 1.67$ & $72.33 \pm 4.86$ & $155.72 \pm 7.49$ & $265.04 \pm 13.98$ \\
\hline & Hongju & $17.00 \pm 0.97$ & $15.18 \pm 0.42$ & $25.91 \pm 0.53$ & $11.44 \pm 1.10$ & $10.82 \pm 1.21$ & $34.26 \pm 2.46$ & $56.52 \pm 2.81$ \\
\hline & Xiangcheng & $14.43 \pm 0.99$ & $16.48 \pm 0.68$ & $18.85 \pm 1.09$ & $10.76 \pm 1.74$ & $26.16 \pm 2.2$ & $52.78 \pm 1.87$ & $89.70 \pm 5.42$ \\
\hline & Zhicheng & $13.97 \pm 0.99$ & $14 \pm 0.75$ & $19.19 \pm 0.96$ & $24.74 \pm 1.55$ & $27.26 \pm 0.97$ & $41.78 \pm 1.70$ & $93.78 \pm 1.34$ \\
\hline & Mean & $15.854 a$ & $14.204 a$ & $22.654 a$ & $21.512 a$ & $30.480 a$ & $79.356 a$ & $131.35 a$ \\
\hline \multirow[t]{2}{*}{ HP } & Cuningmeng & $14.67 \pm 1.43$ & $13.21 \pm 1.06$ & $22.86 \pm 2.03$ & $35.95 \pm 5.83$ & $39.15 \pm 1.74$ & $154.11 \pm 2.88$ & $229.2 \pm 8.79$ \\
\hline & Suanju & $18.27 \pm 1.15$ & $13.32 \pm 0.15$ & $20.64 \pm 0.39$ & $23.59 \pm 4.02$ & $19.12 \pm 1.49$ & $54.78 \pm 4.91$ & $97.49 \pm 10.05$ \\
\hline
\end{tabular}




\begin{tabular}{|c|c|c|c|c|c|c|c|c|}
\hline & Suancheng & $19.13 \pm 1.24$ & $15.16 \pm 0.23$ & $23.99 \pm 0.49$ & $20.72 \pm 2.26$ & $22.10 \pm 2.75$ & $85.90 \pm 6.47$ & $128.73 \pm 7.74$ \\
\hline & Honglimeng & $10.65 \pm 0.45$ & $12.45 \pm 0.53$ & $23.37 \pm 1.19$ & $18.93 \pm 2.57$ & $45.72 \pm 4.45$ & $134.12 \pm 12.16$ & $198.76 \pm 17.80$ \\
\hline & Zhike & $16.00 \pm 2.81$ & $9.85 \pm 0.18$ & $18.89 \pm 0.22$ & $14.19 \pm 3.51$ & $9.95 \pm 0.64$ & $24.59 \pm 1.82$ & $48.73 \pm 5.79$ \\
\hline & Goutoucheng & $13.73 \pm 0.43$ & $12.50 \pm 0.37$ & $22.47 \pm 0.89$ & $8.68 \pm 0.78$ & $16.56 \pm 0.60$ & $47.76 \pm 1.51$ & $73.00 \pm 2.29$ \\
\hline & Xiangyuan & $10.41 \pm 1.35$ & $15.42 \pm 0.87$ & $24.05 \pm 1.74$ & $26.87 \pm 6.65$ & $78.85 \pm 1.89$ & $163.69 \pm 2.00$ & $269.41 \pm 10.32$ \\
\hline & Hongju & $15.17 \pm 0.27$ & $15.12 \pm 0.09$ & $26.13 \pm 0.69$ & $12.49 \pm 1.77$ & $13.82 \pm 0.61$ & $46.43 \pm 3.53$ & $72.75 \pm 5.00$ \\
\hline & Xiangcheng & $13.50 \pm 0.23$ & $14.57 \pm 0.63$ & $16.27 \pm 0.65$ & $13.48 \pm 0.91$ & $34.39 \pm 0.75$ & $61.87 \pm 1.06$ & $109.74 \pm 2.39$ \\
\hline & Zhicheng & $15.63 \pm 0.92$ & $15.24 \pm 0.81$ & $19.22 \pm 0.98$ & $20.26 \pm 0.29$ & $22.60 \pm 1.96$ & $32.19 \pm 3.63$ & $75.05 \pm 5.40$ \\
\hline & Mean & $14.716 b$ & $13.684 a$ & $21.788 a$ & $19.516 a$ & $30.225 a$ & $80.544 a$ & $130.29 a$ \\
\hline \multirow[t]{3}{*}{$\mathrm{F}$ value } & Cultivar(C) & $15.55^{* *}$ & $20.11 * *$ & $13.72 * *$ & $12.01 * *$ & $90.62 * *$ & $121.95^{* *}$ & $86.44 * *$ \\
\hline & Phosphorus (P) & $86.52 * *$ & $51.97 * *$ & $42.32 * *$ & $46.95^{* *}$ & $355.88 * *$ & $447.71 * *$ & $401.89 * *$ \\
\hline & $\mathrm{C} \times \mathrm{P}$ & $3.68 * *$ & $3.29 * *$ & $3.32 * *$ & $4.77 * *$ & $27.40 * *$ & $30.59 * *$ & $27.00 * *$ \\
\hline
\end{tabular}

Note: different lowercase letters are used to indicate values that are significantly different at $p<0.05$ among $P$ treatments.

Table 5. Effects of phosphorus treatment on $M g$ content and $M g$ accumulation in citrus plants.

\begin{tabular}{|c|c|c|c|c|c|c|c|c|}
\hline Phosphorus & Cultivar & $\begin{array}{l}\text { Mg content } \\
\text { in root } \\
\left(\mathbf{m g ~ g}^{-1}\right)\end{array}$ & $\begin{array}{l}\text { Mg content } \\
\text { in branch } \\
\left(\mathrm{mg} \mathrm{g}^{-1}\right)\end{array}$ & $\begin{array}{l}\text { Mg content } \\
\text { in leaves } \\
\left(\mathrm{mg} \mathrm{g}^{-1}\right)\end{array}$ & $\begin{array}{c}\text { Mg } \\
\text { accumulation } \\
\text { in } \operatorname{root}(\mathbf{m g})\end{array}$ & $\begin{array}{c}\text { Mg } \\
\text { accumulation } \\
\text { in branch (mg) }\end{array}$ & \begin{tabular}{l}
\multicolumn{1}{c}{ Mg } \\
accumulation \\
in leaves $(\mathbf{m g})$
\end{tabular} & $\begin{array}{c}\text { Total Mg } \\
\text { accumulation } \\
(\mathrm{mg})\end{array}$ \\
\hline \multirow[t]{9}{*}{ LP } & Cuningmeng & $0.72 \pm 0.06$ & $0.77 \pm 0.07$ & $2.06 \pm 0.16$ & $0.94 \pm 0.12$ & $1.07 \pm 0.15$ & $5.36 \pm 0.93$ & $7.38 \pm 1.11$ \\
\hline & Suanju & $1.26 \pm 0.08$ & $1.92 \pm 0.03$ & $2.74 \pm 0.09$ & $0.75 \pm 0.01$ & $0.79 \pm 0.10$ & $1.91 \pm 0.23$ & $3.45 \pm 0.28$ \\
\hline & Suancheng & $1.23 \pm 0.04$ & $1.14 \pm 0.08$ & $2.27 \pm 0.14$ & $1.61 \pm 0.12$ & $1.12 \pm 0.07$ & $5.43 \pm 0.79$ & $8.16 \pm 0.93$ \\
\hline & Honglimeng & $0.80 \pm 0.02$ & $0.70 \pm 0.04$ & $2.07 \pm 0.09$ & $1.23 \pm 0.07$ & $1.46 \pm 0.11$ & $5.67 \pm 0.42$ & $8.36 \pm 0.59$ \\
\hline & Zhike & $0.98 \pm 0.04$ & $1.57 \pm 0.08$ & $3.32 \pm 0.20$ & $0.74 \pm 0.10$ & $1.24 \pm 0.09$ & $2.97 \pm 0.35$ & $4.96 \pm 0.53$ \\
\hline & Goutoucheng & $1.51 \pm 0.03$ & $0.90 \pm 0.05$ & $2.32 \pm 0.17$ & $1.06 \pm 0.03$ & $0.77 \pm 0.09$ & $2.95 \pm 0.47$ & $4.79 \pm 0.58$ \\
\hline & Xiangyuan & $0.87 \pm 0.05$ & $1.77 \pm 0.11$ & $1.74 \pm 0.09$ & $1.41 \pm 0.17$ & $4.47 \pm 0.52$ & $5.60 \pm 0.58$ & $11.48 \pm 1.21$ \\
\hline & Hongju & $1.21 \pm 0.06$ & $1.05 \pm 0.07$ & $2.17 \pm 0.19$ & $1.24 \pm 0.11$ & $0.41 \pm 0.06$ & $1.93 \pm 0.28$ & $3.58 \pm 0.41$ \\
\hline & Xiangcheng & $0.98 \pm 0.03$ & $1.81 \pm 0.10$ & $1.86 \pm 0.10$ & $1.04 \pm 0.07$ & $1.84 \pm 0.03$ & $3.39 \pm 0.05$ & $6.27 \pm 0.11$ \\
\hline
\end{tabular}




\begin{tabular}{|c|c|c|c|c|c|c|c|c|}
\hline & Zhicheng & $0.49 \pm 0.08$ & $1.54 \pm 0.22$ & $2.50 \pm 0.25$ & $0.84 \pm 0.17$ & $2.05 \pm 0.28$ & $3.57 \pm 0.40$ & $6.46 \pm 0.48$ \\
\hline & Mean & $1.0057 b$ & $1.3173 b$ & $2.3050 a$ & $1.0872 b$ & $1.5219 c$ & $3.8787 c$ & $6.488 c$ \\
\hline \multirow[t]{11}{*}{ MP } & Cuningmeng & $0.97 \pm 0.03$ & $0.74 \pm 0.03$ & $1.85 \pm 0.04$ & $2.21 \pm 0.32$ & $2.96 \pm 0.16$ & $14.31 \pm 0.97$ & $19.49 \pm 1.34$ \\
\hline & Suanju & $1.54 \pm 0.09$ & $1.89 \pm 0.09$ & $2.72 \pm 0.10$ & $1.37 \pm 0.09$ & $1.68 \pm 0.13$ & $4.87 \pm 0.62$ & $7.93 \pm 0.83$ \\
\hline & Suancheng & $1.32 \pm 0.06$ & $1.31 \pm 0.06$ & $2.41 \pm 0.13$ & $2.23 \pm 0.15$ & $2.84 \pm 0.23$ & $10.74 \pm 1.03$ & $15.81 \pm 1.41$ \\
\hline & Honglimeng & $1.12 \pm 0.04$ & $0.71 \pm 0.09$ & $2.37 \pm 0.16$ & $2.14 \pm 0.07$ & $2.43 \pm 0.28$ & $11.57 \pm 0.22$ & $16.14 \pm 0.55$ \\
\hline & Zhike & $1.05 \pm 0.03$ & $1.46 \pm 0.17$ & $3.08 \pm 0.38$ & $1.03 \pm 0.07$ & $1.42 \pm 0.23$ & $3.55 \pm 0.67$ & $6.00 \pm 0.96$ \\
\hline & Goutoucheng & $2.09 \pm 0.13$ & $0.97 \pm 0.08$ & $2.47 \pm 0.13$ & $1.69 \pm 0.23$ & $1.13 \pm 0.05$ & $5.03 \pm 0.20$ & $7.85 \pm 0.41$ \\
\hline & Xiangyuan & $1.54 \pm 0.22$ & $1.88 \pm 0.17$ & $1.88 \pm 0.21$ & $2.98 \pm 0.42$ & $8.05 \pm 0.55$ & $11.1 \pm 1.09$ & $22.13 \pm 1.97$ \\
\hline & Hongju & $1.33 \pm 0.05$ & $1.12 \pm 0.05$ & $2.44 \pm 0.18$ & $0.89 \pm 0.06$ & $0.80 \pm 0.10$ & $3.25 \pm 0.45$ & $4.95 \pm 0.49$ \\
\hline & Xiangcheng & $1.14 \pm 0.07$ & $1.78 \pm 0.11$ & $1.83 \pm 0.12$ & $0.84 \pm 0.12$ & $2.82 \pm 0.29$ & $5.17 \pm 0.52$ & $8.84 \pm 0.75$ \\
\hline & Zhicheng & $0.64 \pm 0.02$ & $1.77 \pm 0.10$ & $2.83 \pm 0.15$ & $1.14 \pm 0.08$ & $3.47 \pm 0.26$ & $6.17 \pm 0.41$ & $10.77 \pm 0.65$ \\
\hline & Mean & $1.2730 a$ & $1.3647 a b$ & $2.3877 a$ & $1.6531 a$ & $2.7599 b$ & $7.5773 b$ & $11.990 \mathrm{~b}$ \\
\hline \multirow[t]{11}{*}{ HP } & Cuningmeng & $1.09 \pm 0.04$ & $0.91 \pm 0.08$ & $2.36 \pm 0.31$ & $2.67 \pm 0.29$ & $2.70 \pm 0.09$ & $15.84 \pm 1.27$ & $21.21 \pm 1.60$ \\
\hline & Suanju & $1.72 \pm 0.03$ & $1.74 \pm 0.01$ & $2.54 \pm 0.02$ & $2.20 \pm 0.26$ & $2.49 \pm 0.19$ & $6.75 \pm 0.63$ & $11.44 \pm 0.97$ \\
\hline & Suancheng & $1.62 \pm 0.18$ & $1.39 \pm 0.02$ & $2.66 \pm 0.08$ & $1.78 \pm 0.32$ & $2.04 \pm 0.32$ & $9.51 \pm 0.67$ & $13.33 \pm 0.8$ \\
\hline & Honglimeng & $1.06 \pm 0.06$ & $0.68 \pm 0.06$ & $2.21 \pm 0.10$ & $1.87 \pm 0.19$ & $2.49 \pm 0.22$ & $12.67 \pm 1.06$ & $17.02 \pm 1.38$ \\
\hline & Zhike & $0.96 \pm 0.13$ & $1.98 \pm 0.09$ & $3.26 \pm 0.45$ & $0.85 \pm 0.19$ & $2.00 \pm 0.16$ & $4.18 \pm 0.39$ & $7.03 \pm 0.38$ \\
\hline & Goutoucheng & $2.19 \pm 0.10$ & $0.88 \pm 0.05$ & $2.13 \pm 0.06$ & $1.39 \pm 0.14$ & $1.17 \pm 0.09$ & $4.55 \pm 0.35$ & $7.11 \pm 0.52$ \\
\hline & Xiangyuan & $1.02 \pm 0.06$ & $1.99 \pm 0.06$ & $1.92 \pm 0.05$ & $2.59 \pm 0.46$ & $10.19 \pm 0.42$ & $13.2 \pm 0.80$ & $25.99 \pm 1.65$ \\
\hline & Hongju & $1.19 \pm 0.01$ & $1.20 \pm 0.06$ & $2.41 \pm 0.11$ & $0.97 \pm 0.11$ & $1.09 \pm 0.06$ & $4.29 \pm 0.38$ & $6.35 \pm 0.52$ \\
\hline & Xiangcheng & $1.29 \pm 0.06$ & $1.63 \pm 0.03$ & $1.66 \pm 0.08$ & $1.30 \pm 0.15$ & $3.87 \pm 0.19$ & $6.36 \pm 0.53$ & $11.52 \pm 0.78$ \\
\hline & Zhicheng & $0.73 \pm 0.05$ & $1.77 \pm 0.08$ & $2.83 \pm 0.17$ & $0.95 \pm 0.08$ & $2.64 \pm 0.24$ & $4.74 \pm 0.54$ & $8.33 \pm 0.71$ \\
\hline & Mean & $1.2860 a$ & $1.4177 a$ & $2.3983 a$ & $1.6557 a$ & $3.0693 a$ & $8.2072 a$ & $12.932 a$ \\
\hline \multirow[t]{3}{*}{ F value } & Cultivar(C) & $48.73^{* *}$ & $116.82 * *$ & $12.72 * *$ & $23.13^{* *}$ & $327.60 * *$ & $47.56^{* * *}$ & $66.01 * *$ \\
\hline & Phosphorus (P) & $41.59^{* *}$ & $2.54^{*}$ & $1.16 \mathrm{~ns}$ & $25.41 * *$ & $105.48 * *$ & $188.53^{* *}$ & $159.23^{* *}$ \\
\hline & $\mathrm{C} \times \mathrm{P}$ & $3.88^{* *}$ & $1.48 \mathrm{~ns}$ & $1.17 \mathrm{~ns}$ & $3.88 * *$ & $11.13 * *$ & $10.52 * *$ & $9.24 * *$ \\
\hline
\end{tabular}

Note: different lowercase letters are used to indicate values that are significantly different at $p<0.05$ among P treatments. 


\section{Correlation analysis}

For all treatments, total dry weight showed significant correlation relationship with root dry weight $(\mathrm{r}=0.8950, P<0.001)$, branch dry weight $(\mathrm{r}=0.9720, P<0.001)$, leaves dry weight $(\mathrm{r}=0.9846, P<0.001), \mathrm{N}$ accumulation in root $(\mathrm{r}=0.9475, P<0.001), \mathrm{N}$ accumulation in branch $(\mathrm{r}=0.8796, P<0.001), \mathrm{N}$ accumulation in leaves $(\mathrm{r}=0.9684$, $P<0.001)$, total $\mathrm{N}$ accumulation ( $\mathrm{r}=0.9724, P<0.001), \mathrm{P}$ accumulation in root $(0.7906$, $P<0.001), \mathrm{P}$ accumulation in branch $(0.8581, P<0.001), \mathrm{P}$ accumulation in leaves (0.9244 $P<0.001)$, total $\mathrm{P}$ accumulation $(0.9070, P<0.001), \mathrm{K}$ accumulation in root (0.7764, $P<0.001)$, K accumulation in branch $(0.9414, P<0.001)$, accumulation in leaves $(0.9717, P<0.001)$, total $\mathrm{K}$ accumulation $(0.9790, P<0.001), \mathrm{Mg}$ accumulation in root $(0.8365, P<0.001), \mathrm{Mg}$ accumulation in branch $(0.7293, P<0.001), \mathrm{Mg}$ accumulation in leaves $(0.9488, P<0.001)$, total $\mathrm{mg}$ accumulation $(0.9699, P<0.001)$. Similar correlation relationships were observed for MP and HP treatment. However, for LP, there investigated no significant correlation relationship between total dry weight and $\mathrm{P}, \mathrm{K}, \mathrm{Mg} \mathrm{P}$ accumulation in root. Significant negative correlation relationship between total dry weight and $\mathrm{N}$ content in branch was found. Significant negative correlation relationship between total dry weight and $\mathrm{N}$ content in leaves was observed for MP and HP only. Significant negative correlation relationship between total dry weight and $\mathrm{P}$ content in root was observed for LP. There investigated significant negative correlation relationship between total dry weight and $\mathrm{P}$ content in branch for LP, MP and HP. Significant negative correlation relationship between total dry weight and $\mathrm{K}$ content in root was observed for LP and HP. Total dry weight showed significant negative correlation with $\mathrm{Mg}$ content in leaves for all treatment, LP and MP (Table 6).

\section{Discussion}

In this study, we observed significant effects of rootstock on root dry weight, branch dry weight, leaves dry weight, and total dry weigh (Table 1). Similar to many previous reports (Li and Zhang, 2008; Shafieizargar et al., 2012; Cantuarias-Avilés et al., 2010; 2011; Tazima et al., 2013). Rootstock affects the horticultural characteristics in citrus (Cantuarias-Avilés et al., 2010; Zhang et al., 2011; Hussain et al., 2013; Benjamin et al., 2013; Simpson et al., 2014). The P efficiency is defined as the ability of the plant growth under low effective P concentration condition (Zhang, 1993). Researcher used the plant dry weight to evaluate the plant resistance to low phosphorus stress (Cao et al., 2000; Zhang et al., 2005; Zhou et al., 2005). Here, we found significant effects of phosphorus on $\mathrm{N}, \mathrm{P}, \mathrm{K}$ and $\mathrm{Mg}$ content, and their accumulation in plant (Table 2-5). It confirmed that it could have significant differences in responses of citrus trees to $\mathrm{P}$ fertilization (Wutscher, 1989; Mattos et al., 2006; Fan and Luo, 2015). Previously it was reported that different citrus trees respond to $\mathrm{P}$ differently due to the difference in the acid phosphatase activity of rhizosphere soil, for various factors affecting the acid phosphatase activity (Bonmati et al., 1991; Oberson et al., 1993; Luo and Fan, 2014). 
Table 6. Correlation relationship between total dry weight and the investigated parameters.

\begin{tabular}{|c|c|c|c|c|c|c|c|c|}
\hline \multirow[b]{2}{*}{ Investigated parameters } & \multicolumn{2}{|c|}{ All treatment } & \multicolumn{2}{|c|}{$\mathbf{L P}$} & \multicolumn{2}{|c|}{ MP } & \multicolumn{2}{|c|}{ HP } \\
\hline & $\begin{array}{c}\text { Correlation } \\
\text { coefficient }\end{array}$ & $P$ value & $\begin{array}{c}\text { Correlation } \\
\text { coefficient }\end{array}$ & $P$ value & $\begin{array}{c}\text { Correlation } \\
\text { coefficient }\end{array}$ & $P$ value & $\begin{array}{c}\text { Correlation } \\
\text { coefficient }\end{array}$ & $P$ value \\
\hline Root dry weight & 0.8950 & 0.0000 & 0.8650 & 0.0012 & 0.9101 & 0.0003 & 0.9319 & 0.0001 \\
\hline Branch dry weight & 0.9720 & 0.0000 & 0.9584 & 0.0000 & 0.9790 & 0.0000 & 0.9591 & 0.0000 \\
\hline Leaves dry weight & 0.9846 & 0.0000 & 0.9658 & 0.0000 & 0.9857 & 0.0000 & 0.9841 & 0.0000 \\
\hline $\mathrm{N}$ content in root & 0.1028 & 0.5888 & -0.0982 & 0.7873 & -0.0712 & 0.8451 & -0.2058 & 0.5685 \\
\hline $\mathrm{N}$ content in Branch & -0.4494 & 0.0127 & -0.6534 & 0.0405 & -0.8189 & 0.0038 & -0.9109 & 0.0002 \\
\hline $\mathrm{N}$ content in leaves & -0.2151 & 0.2536 & -0.0400 & 0.9127 & -0.7052 & 0.0227 & -0.7310 & 0.0163 \\
\hline $\mathrm{N}$ accumulation in root & 0.9475 & 0.0000 & 0.7889 & 0.0067 & 0.9423 & 0.0000 & 0.9721 & 0.0000 \\
\hline $\mathrm{N}$ accumulation in branch & 0.8796 & 0.0000 & 0.8917 & 0.0005 & 0.8538 & 0.0017 & 0.8728 & 0.0010 \\
\hline $\mathrm{N}$ accumulation in leaves & 0.9674 & 0.0000 & 0.9723 & 0.0000 & 0.9790 & 0.0000 & 0.9846 & 0.0000 \\
\hline Total $\mathrm{N}$ accumulation & 0.9724 & 0.0000 & 0.9815 & 0.0000 & 0.9769 & 0.0000 & 0.9927 & 0.0000 \\
\hline $\mathrm{P}$ content in root & 0.1221 & 0.5203 & -0.7387 & 0.0147 & -0.5794 & 0.0792 & -0.6262 & 0.0527 \\
\hline $\mathrm{P}$ content in Branch & 0.0446 & 0.8148 & -0.7919 & 0.0063 & -0.7098 & 0.0215 & -0.7050 & 0.0228 \\
\hline $\mathrm{P}$ content in leaves & 0.3448 & 0.0621 & -0.2859 & 0.4232 & -0.1991 & 0.5813 & 0.0998 & 0.7837 \\
\hline $\mathrm{P}$ accumulation in root & 0.7906 & 0.0000 & 0.6066 & 0.0630 & 0.9293 & 0.0001 & 0.7646 & 0.0100 \\
\hline $\mathrm{P}$ accumulation in branch & 0.8581 & 0.0000 & 0.7685 & 0.0094 & 0.9303 & 0.0001 & 0.9410 & 0.0000 \\
\hline $\mathrm{P}$ accumulation in leaves & 0.9244 & 0.0000 & 0.9719 & 0.0000 & 0.9816 & 0.0000 & 0.9771 & 0.0000 \\
\hline Total $\mathrm{P}$ accumulation & 0.9070 & 0.0000 & 0.9396 & 0.0001 & 0.9857 & 0.0000 & 0.9834 & 0.0000 \\
\hline $\mathrm{K}$ content in root & -0.0701 & 0.7129 & -0.8036 & 0.0051 & -0.2148 & 0.5513 & -0.6357 & 0.0482 \\
\hline
\end{tabular}




\begin{tabular}{|c|c|c|c|c|c|c|c|c|}
\hline $\mathrm{K}$ content in Branch & 0.2894 & 0.1208 & -0.1185 & 0.7444 & 0.1328 & 0.7145 & 0.2188 & 0.5437 \\
\hline $\mathrm{K}$ content in leaves & 0.3703 & 0.0440 & -0.1351 & 0.7097 & 0.2725 & 0.4462 & 0.2653 & 0.4588 \\
\hline $\mathrm{K}$ accumulation in root & 0.7764 & 0.0000 & 0.3851 & 0.2718 & 0.8299 & 0.0030 & 0.7066 & 0.0223 \\
\hline $\mathrm{K}$ accumulation in branch & 0.9414 & 0.0000 & 0.9342 & 0.0001 & 0.9316 & 0.0001 & 0.9332 & 0.0001 \\
\hline $\mathrm{K}$ accumulation in leaves & 0.9717 & 0.0000 & 0.9587 & 0.0000 & 0.9788 & 0.0000 & 0.9721 & 0.0000 \\
\hline Total $\mathrm{K}$ accumulation & 0.9790 & 0.0000 & 0.9934 & 0.0000 & 0.9811 & 0.0000 & 0.9903 & 0.0000 \\
\hline $\mathrm{Mg}$ content in root & -0.1220 & 0.5206 & -0.5616 & 0.0911 & -0.2087 & 0.5628 & -0.2887 & 0.4185 \\
\hline $\mathrm{Mg}$ content in Branch & -0.1617 & 0.3934 & -0.2026 & 0.5746 & -0.3008 & 0.3983 & -0.1497 & 0.6798 \\
\hline $\mathrm{Mg}$ content in leaves & -0.4858 & 0.0065 & -0.6563 & 0.0393 & -0.6525 & 0.0408 & -0.5292 & 0.1157 \\
\hline $\mathrm{Mg}$ accumulation in root & 0.8365 & 0.0000 & 0.5384 & 0.1084 & 0.8343 & 0.0027 & 0.8284 & 0.0031 \\
\hline $\mathrm{Mg}$ accumulation in branch & 0.7293 & 0.0000 & 0.7295 & 0.0166 & 0.6554 & 0.0396 & 0.7234 & 0.0180 \\
\hline $\mathrm{Mg}$ accumulation in leaves & 0.9488 & 0.0000 & 0.9224 & 0.0001 & 0.9730 & 0.0000 & 0.9304 & 0.0001 \\
\hline Total $\mathrm{Mg}$ accumulation & 0.9699 & 0.0000 & 0.9643 & 0.0000 & 0.9683 & 0.0000 & 0.9785 & 0.0000 \\
\hline
\end{tabular}


$\mathrm{P}$ is an important for plant growth, development and reproduction as it is an essential constituent of phospholipids, nucleic acid and many proteins. With increasing $\mathrm{P}$ application, the root, branch and leaves dry weight, and total dry weight increased, however different cultivars responded differently (Table 1-5). Low P availability in soil is one of the most critical aspects that limit the productivity of many crops (Sanchez and Salinas, 1981). P deficiency or excess phosphate fertilization could cause nutrient imbalance in citrus tree and reduce the yield and fruit quality (Fan and Wang, 2012; Fan and Luo, 2015). When the phosphorus nutrition level was extremely low or high, $\mathrm{P}, \mathrm{Mg}$, $\mathrm{Mn}$ and $\mathrm{Cu}$ content was deficient or excessively high and inhibited absorption of $\mathrm{N}, \mathrm{K}$, $\mathrm{Fe}, \mathrm{Zn}$, and B (Fan and Wang, 2012). In our study, we found that low P treatment affected the P, K, and Mg accumulation in root (Table 3-6). Moreover, Syvertsen (1987) reported that $\mathrm{P}$ level in leaves showed no relationship with photosynthetic in orange and pomelo seedlings. Bernardi et al. (2015) reported that $\mathrm{P}$ had little interference on photosynthesis. Zambrosi et al. (2013a) indicated that phosphorus uptake by young citrus plants in low-P soil depends on rootstock varieties and nutrient management. Therefore in our work, low P treatment may affected $\mathrm{Mg}$ content in leaves may lead to low plant photosynthesis and finally decreased total dry weight (Tables 5 and 6). Further study to investigate the improvement citrus rootstocks growth under low $\mathrm{P}$ condition by $\mathrm{Mg}$ application is need.

\section{Conclusion}

In crux, $\mathrm{P}$ application increased $\mathrm{N}, \mathrm{P}, \mathrm{K}$ and $\mathrm{Mg}$ accumulation in citrus plants. With increasing $\mathrm{P}$ application, the plant total plant dry biomass was increased. Low $\mathrm{P}$ treatment affected $\mathrm{P}, \mathrm{K}$, and $\mathrm{Mg}$ accumulation in root and ultimately plant growth. Moreover, low P treatment may affect $\mathrm{Mg}$ content in leaves that may lead to low plant photosynthesis and finally decreased total dry weight.

Acknowledgements. This work was supported by the National Natural Science Foundation (project no. 31372008), Agriculture Fund (no. 2014A020208085, 2014A020209081, 2015A020209110 and 2016LM3174) in Guangdong Province, National Key Research and Development Planning Project (2016YFD0200404-1) and National Science and Technology Planning Project (2013BAD05B04). The authors would like to thank Dr. Zhaowen Mo and Umair Ashraf for their contributions to the manuscript.

\section{REFERENCE}

[1] Benjamin, G., Tietel, Z., Porat, R. (2013): Effects of rootstock/scion combinations on the flavor of citrus fruit. - Journal of Agricultural and Food chemistry 61(47): 11286-11294.

[2] Bernardi, A. C. D. C., Carmello, Q. A. D. C., Carvalho, S. A. D., Machado, E. C., Medina, C. L., Gomes, M. D. M. D. A., Lima, D. M. (2015): Nitrogen, phosphorus and potassium fertilization interactions on the photosynthesis of containerized citrus nursery trees. - Journal of Plant Nutrition 38(12): 1902-1912.

[3] Bonmati, M., Ceccanti, B., Nanniperi, P. (1991): Spatial variability of phosphatase, urease, protease, organic carbon and total nitrogen in soil. - Soil Biology and Biochemistry 23(4): 391-396.

[4] Cantuarias-Aviles, T., Mourão Filho, F. D. A. A., Stuchi, E. S., da Silva, S. R., EspinozaNúñez, E. (2010): Tree performance and fruit yield and quality of 'okitsu' satsuma mandarin grafted on 12 rootstocks. - Scientia Horticulturae 123(3): 318-322. 
[5] Cantuarias-Avilés, T., Mourão Filho, F. D. A. A., Stuchi, E. S., da Silva, S. R., EspinozaNuñez, E. (2011): Horticultural performance of 'folha murcha' sweet orange onto twelve rootstocks. - Scientia Horticulturae 129(2): 259-265.

[6] Cao, J., Zhang, F. S. (2000): Phosphorus uptake and utilization efficiency in seedlings of different wheat genotypes as influenced by water supply at low soil phosphorus availability. - Acta Phytoecologica Sinica 24(6): 731-735. (in Chinese)

[7] Fan, W. G., Luo, Y. (2015): Growth status, root morphology and physiological characteristics of four citrus rootstocks under different phosphorus levels. - Scientia Agricultura Sinica 48(3): 534-545. (in Chinese with English abstract)

[8] Fan, W. G., Wang, L. X. (2012): Effect of different phosphorus levels on growth and leaf element contents of young new hall navel orange trees. - Scientia Agricultura Sinica 45(4): 714-725. (in Chinese with English abstract)

[9] Gimeno, V., Syvertsen, J. P., Rubio, F., Martínez, V., García-Sánchez, F. (2010): Growth and mineral nutrition are affected by substrate type and salt stress in seedlings of two contrasting citrus rootstocks. - Journal of Plant Nutrition 33(10): 1435-1447.

[10] Hussain, S., Curk, F., Anjum, M. A., Pailly, O., Tison, G. (2013): Performance evaluation of common clementine on various citrus rootstocks. - Scientia Horticulturae 150: 278282.

[11] Li, T. Z., Zhang, Z. H. (2008): Modern fruit tree biology. - Beijing Science Press, Beijing, 130-134. (In Chinese)

[12] Liao, H., Yan, X. L. (2003): Advanced plant nutrition. - Beijing Science Press, Beijing, 152-153.

[13] Lu, R. K. (1999): Analys is measures for agro-chemical on soil. - China Agricultural Science and Technology Press, Beijing, 1-638 (In Chinese)

[14] Luo, Y., Fan, W. G. (2014): Organic acid content, microbial quantity and enzyme activity in rhizosphere soil of four citrus rootstocks under different phosphorus levels. - Scientia Agricultura Sinica 47 (5): 955-967.

[15] Mattos Jr, D., Quaggio, J. A., Cantarella, H., Alva, A. K., Graetz, D. A. (2006): Response of young citrus trees on selected rootstocks to nitrogen, phosphorus, and potassium fertilization. - Journal of Plant Nutrition 29(8): 1371-1385.

[16] Oberson, A., Fardeau, J. C., Besson, J. M., Sticher, H. (1993): Soil phosphorus dynamics in cropping systems managed according to conventional and biological agricultural methods. - Biology and Fertility of Soils 16(2): 111-117.

[17] Pestana, M., de Varennes, A., Abadía, J., Faria, E. A. (2005): Differential tolerance to iron deficiency of citrus rootstocks grown in nutrient solution. - Scientia Horticulturae 104(1): 25-36.

[18] Sánchez, P. A., Salinas, J. G. (1981): Low-input technology for managing oxisols and ultisols in tropical America. - Advances in Agronomy 34: 279-406.

[19] Shafieizargar, A., Awang, Y., Juraimi, A. S., Othman, R. (2012): Yield and fruit quality of 'queen' orange [Citrus sinensis (L) Osb.] grafted on different rootstocks in Iran. Australian Journal of Crop Science 6(5): 777.

[20] Simpson, C. R., Nelson, S. D., Melgar, J. C., Jifon, J., King, S. R., Schuster, G., Volder, A. (2014): Growth response of grafted and ungrafted citrus trees to saline irrigation. Scientia Horticulturae 169: 199-205.

[21] Syvertsen, J. P. (1987): Nitrogen content and $\mathrm{CO}_{2}$ assimilation characteristics of citrus leaves. - HortScience 22: 289-291.

[22] Tazima, Z. H., Neves, C. S. V. J., Yada, I. F. U., Leite Júnior, R. P. (2013): Performance of 'okitsu' satsuma mandarin on nine rootstocks. - Scientia Agricola 70(6): 422-427.

[23] Wutscher, H. K. (1989): Alteration of fruit tree nutrition through rootstocks. HortScience 24:578-584.

[24] Zambrosi, F. C. B., Mattos Jr, D., Quaggio, J. A., Cantarella, H., Boaretto, R. M. (2013a): Phosphorus uptake by young citrus trees in low-P soil depends on rootstock varieties and 
nutrient management. - Communications in Doil Science and Plant Analysis 44(14): 2107-2117.

[25] Zambrosi, F. C. B., Mesquita, G. L., Tanaka, F. A. O., Quaggio, J. A., Mattos, D. (2013b): Phosphorus availability and rootstock affect copper-induced damage to the root ultra-structure of citrus. - Environmental and Experimental Botany 95: 25-33.

[26] Zhang, F. S. (1993) Ecological Physiology and genetics of plant nutrition. - China Agricultural University Press, Beijing: 1-13, 18. (in Chinese)

[27] Zhang, L. M., He, L. Y., Li, J. S., Xu, S. Z. (2005) Phosphorus nutrient characteristics of different maize inbreds with tolerance to low-P stress. - Scientia Agricultura Sinica 38(1): 110-115. (in Chinese)

[28] Zhang, X., Breksa III, A. P., Mishchuk, D. O., Slupsky, C. M. (2011): Elevation, rootstock, and soil depth affect the nutritional quality of mandarin oranges. - Journal of Agricultural and Food Chemistry 59 (6): 2672-2679.

[29] Zhou, Z. C., Xie, Y. R., Jin, G. Q., Chen, Y., Song, Z. Y. (2005): Study on phosphorus efficiency of different provenances of pinus massoniana. - Scientia silvae sinicae 7 , 41(4): 25-30. (in Chinese) 